\title{
Vibration of composite thin-walled beams with variable open cross section by a high order implicit algorithm
}

\author{
A. EL Kaimbillah' ${ }^{1}$, O. Bourihane ${ }^{1,2}$, M. Jamal ${ }^{1}$, B. Braikat ${ }^{1}$, F. Mohri ${ }^{3}$, N. Damil ${ }^{1}$ \\ 1. Laboratoire d'Ingénierie et Matériaux (LIMAT), Faculté des Sciences Ben M'Sik, Hassan II University of Casablanca, \\ Avenue Cdt Driss El Harti B.P. 7955, Sidi Othman, Casablanca, Maroc. a.elkaimbillah@gmail.com, \\ jamalmoham@gmail.com,b.braikat@gmail.com,noureddine.damil@gmail.com \\ 2. Laboratoire de Génie Mécanique (LGM), Faculté des Sciences et Techniques, Fès, Université Sidi Mohamed Ben \\ Abdellah Route d'Imouzzer B.P. 2202, Fès, Maroc. o.bourihane@gmail.com \\ 3. Laboratoire d'Etude des Microstructures et de Mécanique des Matériaux (LEM3), Université de Lorraine Metz, \\ CNRS UMR 7239, Ile du Saulcy, 57057, France. foudil.mohri@univ-lorraine.fr
}

\begin{abstract}
:
In this work, we study the forced nonlinear vibrations with large amplitude and large torsion of composite thinwalled beams with open variable cross sections under external dynamic loads using a high order implicit algorithm. The used algorithm is based on the temporal and spatial discretizations, the homtopoy transformation, Taylor series expansion and the continuation technique. A 3D beam element with two nodes and seven degrees of freedom per node is adopted. The obtained results are compared with those computed by the industriel Abaqus code.
\end{abstract}

Keywords: Composite thin-walled beam, open variable cross section, large displacement, large torsion, forced nonlinear vibrations, Newmark implicit scheme, Taylor series, homotopy transformation.

\section{Introduction}

For about a century, many industrial sectors have sought to strengthen and at the same time lighten the structural solutions to optimize the efficiency and cost of structures. Among them are the civil, mechanical, naval and aerospace industries. This has led to an increasing use of thin-walled open-section structures such as cold-formed steel girders, steel and concrete girders, ship hulls, trapezoidal steel sheets and other structures in which a dimension is small compared to other dimensions. The use of rigid beams that have a slight weight encourages industrials to use composite beams with open and variable sections.

To ensure the use of thin-walled composite beams, it is important to study their behavior when it is subjected to external dynamic loading. Several efforts have been devoted to this research domain. Many finite element models have been proposed to study the linear and nonlinear dynamic responses of these thin-walled structures with constant and variable open sections under these external loadings.

This work deals with the study of forced nonlinear vibrations with large amplitude and large torsion of composite thin-walled beam with open variable cross sections under external dynamic loads. The free vibrations of composite beams have been studied in [1] using the thin tube theory for analyzing thin-walled composite beams. This study is focused on an analysis of free vibrations of a composite thin-walled beam with open and variable cross section. We propose in this work to study the nonlinear forced vibration of this structures type. The influence of the section and the material effect on the vibrational behavior of thin-walled beam is also studied. The nonlinear dynamic behavior of this structures type is investigated by a high order implicit algorithm [2]. This algorithm combines the discretization procedures in space and time respectively, homotopy technique, Taylor series representation and a continuation procedure.

\section{Governing dynamic equations and reso- lution strategy}

Consider a composite $3 D$ thin-walled beam of length $L$ and open variable cross section of area $A(x)$. The used rectangular cartesian co-ordinates system is Gxyz with origin at $G, G_{y}$ and $G_{z}$ are the principal bending axes and $G_{x}$ is the axial axis (see figure 1). The co-ordinates of shear point $C$ located in $G y z$ plane are $y_{c}, z_{c}$. An arbitrary point $M$ of a section is defined by its coordinates $(y, z, \omega)$; with $\omega$ is the sectoriel coordinate. The displacement components $u_{M}, v_{M}$ and $w_{M}$ at point $M$ are expressed by the following relations [3]: 


$$
\left\{U_{M}\right\}=\left\{\begin{aligned}
u_{M} & =u-y \alpha_{z}-z \alpha_{y}-\omega(y, z) \theta_{x}^{\prime} \\
v_{M} & =v-\left(z-z_{c}\right) s+\left(y-y_{c}\right) c \\
w_{M} & =w+\left(y-y_{c}\right) s+\left(z-z_{c}\right) c
\end{aligned}\right.
$$

with:

$$
\left\{\begin{array}{lll}
c=\cos \left(\theta_{x}\right)-1 & , & s=\sin \left(\theta_{x}\right) \\
\alpha_{y}=(1+c) w^{\prime}-v^{\prime} s & , & \alpha_{z}=(1+c) v^{\prime}+w^{\prime} s
\end{array}\right.
$$

where $u$ represents the axial displacement of $G$, the transversal components $v$ and $w$ represent the displacements of shear point $C$ in $y$ and $z$ directions and $\theta_{x}$ is the torsion angle.

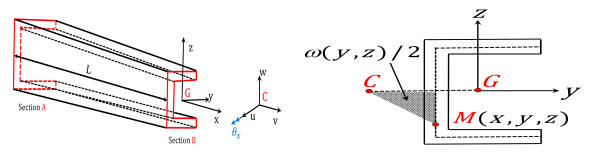

Figure 1: Thin-walled variable open section beam and coordinates of the point $M$ on the cross section

The governing dynamic equations are obtained from the principle of virtual work: $\delta\left(T+U-W_{e x t}\right)=0$; with $\delta U$ is the virtual strain energy, $\delta T$ is the virtual kinetic energy and $\delta W_{\text {ext }}$ is the virtual work of the external forces. The discretization of governing dynamic equations by the finite element method using a $3 D$ finite element beam with two nodes and seven degrees of freedom per node are given by:

$$
[M]\{\ddot{r}\}+[C(\dot{\theta}, \dot{\theta})]\{\dot{r}\}+[K(\theta, \alpha)]\{r\}=\left\{F_{\text {ext }}\right\}
$$

where $[M]$ is the mass matrix, $[C(\dot{\theta}, \dot{\theta})]$ is the damping matrix, $[K(\theta, \alpha)]$ is the stiffness matrix and $\left\{F_{\text {ext }}\right\}$ is the applied load vector. To solve the nonlinear dynamic equations (3) we adopt the high order algorithm presented in [3].

\section{Numerical applications and discussions}

To investigate the influence of composite sections on the behavior of the thin-walled beam and also to show the importance of using composite thin-walled beams with variable open cross section, we analyze the dynamical behavior of this beam for two cases: the first concerns the free vibration and the second is dedicated to the nonlinear forced vibration.

\subsection{Linear case}

In this section, we study the linear free vibration of compsite thin-walled beam with variable open cross section. Indeed we consider a clamped-free bi-symmetrical composite beam of length $L=40 \mathrm{~cm}$, of an I-section varying in web height and flange width linearly, while all other dimensions remain constant (see figure 2-(a)). All numerical analyses performed in this section are the same data used in [1]. The geometric characteristics of the extreme end sections of the beam are shown in the figure 2-(b). The beam is composed of two material types: flange and web. The mechanical characteristics of the web are $E_{w}=7.010^{3} \mathrm{KN} / \mathrm{cm}^{2}, G_{w}=2.610^{3} \mathrm{KN} / \mathrm{cm}^{2}$ and $\rho_{w}=2.85 t / \mathrm{m}^{3}$ and the mechanical characteristics of the flange are: $E_{f}=2.110^{4} \mathrm{KN} / \mathrm{cm}^{2}, G_{f}=$ $8.0510^{3} \mathrm{KN} / \mathrm{cm}^{2}$ and $\rho_{f}=7.85 t / \mathrm{m}^{3}$. The considered beam is meshed in $203 D$ beam elements. In Table (1), the ten first eigenfrequencies of the free vibrating beam are presented and compared with those obtained by the industrial Abaqus code and those given in [1].

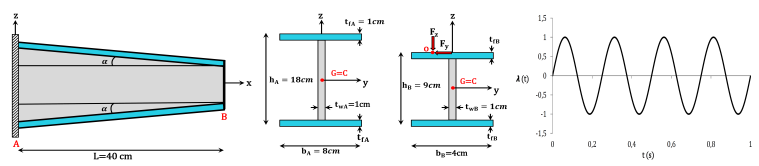

Figure 2: Geometrical characteristics of a I section composite thin-walled beam with variable open cross section and temporal evolution of external dynamic load

\begin{tabular}{lccc}
\hline$\omega_{i}$ & {$[1]$} & present study & Abaqus \\
\hline 1 & 282.5493 & 284.1088 & 285.3457 \\
\hline 2 & 974.6288 & 979.8491 & 981.7391 \\
\hline 3 & 2244.756 & 2254.054 & 2255.276 \\
\hline 4 & 4127.814 & 4132.001 & 4132.765 \\
\hline 5 & 6633.401 & 6640.321 & 6641.566 \\
\hline 6 & 9762.938 & 9765.432 & 9766.941 \\
\hline 7 & 13516.330 & 13519.899 & 13520.189 \\
\hline 8 & 17892.780 & 17895.911 & 17896.744 \\
\hline 9 & 22891.272 & 22896.100 & 22897.243 \\
\hline 10 & 28510.183 & 28512.002 & 28512.581 \\
\hline
\end{tabular}

Table 1: Comparison of eigenfrequencies $\omega_{i}$ of the composite beam

According to the table (1), we notice that the results are in good agreement with those calculated by the industrial Abaqus code and those given in [1].

\subsection{Nonlinear case}

In order to study the forced nonlinear vibrations of composite thin-walled beam with variable open cross sec- 
tion, we consider the same beam studied in (section 3.1). This beam is subjected to two temporal eccentric loadings $F_{y}^{o}(t)$ and $F_{z}^{o}(t)$ applied at point $O$; with $F_{z}^{o}(t)=$ $600 K N \lambda(t), F_{y}^{o}(t)=100 K N \lambda(t) \lambda(t)$ is that plotted in figure 2-(c), eccentricities of these loadings are: $e_{y}=-0.5 \mathrm{~cm}$ and $e_{z}=4.5 \mathrm{~cm}$. Firstly, we study the influence of the variation of the section on the forced nonlinear vibrations behavior. To do this, we take three values $\alpha_{1}=0^{\circ}, \alpha_{2}=32.0054^{\circ}$ and $\alpha_{3}=48.3665^{\circ}$ with the corresponding heights $h_{A 1}=9 \mathrm{~cm}, h_{A 2}=14 \mathrm{~cm}$, $h_{A 3}=18 \mathrm{~cm}$ and $h_{B}=9 \mathrm{~cm}$. The nonlinear dynamic analysis is made in the time range $[0,1 s]$ with a time step $\Delta t=10^{-3} \mathrm{~s}$. The problem have been solved by the high order implicit algorithm with a truncation order $p=12$ and a tolerance parameter $\varepsilon=10^{-6}$. The obtained results are compared with those obtained by the industrial Abaqus code, using $(30 \times 2 \times 3=180)$ 3D-Shell element « S8R » and with the same time step. The figure (3) presents the temporal evolution of the torsion angle component $\theta_{x}$ for different values of $\alpha$.

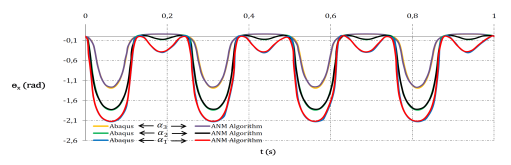

Figure 3: Temporal evolution of the component $\theta_{x}$

The obtained results are in good agreement with those calculated by the industrial Abaqus code. In addition, this study shows that the axial variation of the open cross section achieved by varying the values of the angle $\alpha$ which has a significant influence on the dynamic responses of the thin-walled beam. Secondly, we study the effect of composite section on the dynamic response of composite thiw-walled beam with open variable cross section. For this, the study is analyzed with the ration $r i=E_{f} / E_{w}$ for a fixed value of $E_{w}=70 G p a$.

\begin{tabular}{c|c|c|c|c|c|c}
\hline \multirow{2}{*}{$r_{i}$} & \multicolumn{3}{|c|}{ Flange } & \multicolumn{3}{c}{ Web } \\
\cline { 2 - 7 } & $E_{f}$ & $\nu_{f}$ & $\rho_{f}$ & $E_{W}$ & $\nu_{W}$ & $\rho_{W}$ \\
\hline$r_{1}=1$ & 70 & 0.34 & 2.85 & 70 & 0.34 & 2.85 \\
\hline$r_{2}=3$ & 210 & 0.3 & 7.85 & 70 & 0.34 & 2.85 \\
\hline$r_{3}=5$ & 350 & 0.25 & 3.95 & 70 & 0.34 & 2.85 \\
\hline$r_{4}=0.71$ & 50 & 0.3 & 1.8 & 70 & 0.34 & 2.85 \\
\hline
\end{tabular}

Table 2: Values of mechanical characteristics of used materials

The figure (4) shows the temporal evolutions of the displacements components $u, v, w$ and $\theta_{x}$ of composite thinwalled beam with open variable cross section.

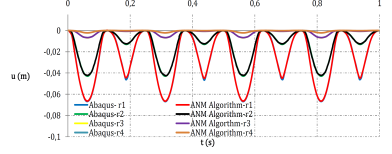

(a) Temporal evolutions of $u$

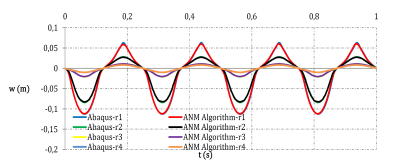

(c) Temporal evolutions of $w$

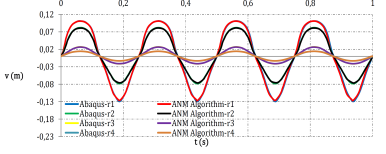

(b) Temporal evolutions of $v$
Figure 4: Dynamical response of thin-walled composite with open variable cross section for different values of ratio $r_{i}$

According to figure (4), we note that the obtained results are in a good agreement with those calculated by the industrial Abaqus code. On the other hand, we remark that when the ratio ri increases the effect becomes important and the amplitude decreases. In addition, we notice that if the material used in the flange is more rigid than the other used in the web the amplitude decreases and the beam becomes more rigid and in contrary if the material of the web is more rigid. The influence of composite sections is remarkable, thus, the amplitude of the forced vibrations decreases in a considerable manner.

\section{Conclusion}

This work is dedied to investigate numerically the behavior dynamic of thin-walled composite beams with open variable cross section subjected to external arbitrary loadings by the high order implicit algorithm. The obtained results are compared with those calculated by the industrial Abaqus code. Indeed this comparison shows that these results are consistent and illustrate the performance of this algorithm.

\section{References}

[1] E. J. Sapountzakis, Torsional vibrations of composite bars of variable cross-section by BEM, Computer Methods in Applied Mechanics and Engineering 194 (2005) 2127-2145.

[2] M. Jamal, B. Braikat, S. Boutmir, N. Damil, M. Potier-Ferry, A high order implicit algorithm for solving instationary non-linear problems, Computational Mechanics 28 (2002) 375-380.

[3] A. El Kaimbillah, O. Bourihane, B. Braikat, M. Jamal, F. Mohri, N. Damil, Efficient high-order implicit solvers for the dynamic of thin-walled beams with open cross section under external arbitrary loadings, Discrete \& Continuous Dynamical Systems-S 12 (2019) 2867-2885. 